\title{
Smad3 Suppresses Epithelial Cell Migration and Proliferation via the Clock Gene Dec1, Which Negatively Regulates the Expression of Clock Genes Dec2 and Per1
}

\author{
Fuyuki Sato, ${ }^{\star}$ Tsuyoshi Otsuka ${ }^{\dagger}$ Akira Kohsaka, ${ }^{\dagger}$ Hue Thi Le, ${ }^{\dagger}$ Ujjal K. Bhawal, ${ }^{\ddagger}$ and Yasuteru Muragaki*
}

From the Departments of Pathology* and Physiology, ${ }^{\dagger}$ Wakayama Medical University School of Medicine, Wakayama; and the Department of Biochemistry and Molecular Biology, ${ }^{\ddagger}$ Nihon University School of Dentistry at Matsudo, Chiba, Japan

\author{
Accepted for publication \\ January 2, 2019. \\ Address correspondence to \\ Fuyuki Sato, Ph.D., D.D.S., \\ Department of Pathology, \\ Wakayama Medical University \\ School of Medicine, 811-1 \\ Kimiidera, Wakayama 641- \\ 8509, Japan. E-mail: satof@ \\ wakayama-med.ac.jp.
}

\begin{abstract}
Smad3 has circadian expression; however, whether Smad3 affects the expression of clock genes is poorly understood. Here, we investigated the regulatory mechanisms between Smad3 and the clock genes Dec1, Dec2, and Per1. In Smad3 knockout mice, the amplitude of locomotor activity was decreased, and Dec1 expression was decreased in the suprachiasmatic nucleus, liver, kidney, and tongue compared with control mice. Conversely, Dec2 and Per1 expression was increased compared with that of control mice. In Smad3 knockout mice, immunohistochemical staining revealed that Dec1 expression decreased, whereas Dec2 and Per1 expression increased in the endothelial cells of the kidney and liver. In NIH3T3 cells, Smad3 overexpression increased Dec1 expression, but decreased Dec2 and Per1 expression. In a wound-healing experiment that used Smad3 knockout mice, Dec1 expression decreased in the basal cells of squamous epithelium, promoting wound healing of the mucosa. Finally, the migration and proliferation of Smad3 knockdown squamous carcinoma cells was suppressed by Dec1 overexpression but was promoted by Dec2 overexpression. Dec1 overexpression decreased E-cadherin and proliferating cell nuclear antigen expression, whereas these expression levels were increased by Dec2 overexpression. These results suggest Smad3 is relevant to circadian rhythm and regulates cell migration and proliferation through Dec1, Dec2, and Per1 expression. (Am J Pathol 2019, 189: 773-783; https://doi.org/10.1016/j.ajpath.2019.01.006)
\end{abstract}

Circadian rhythms are dominantly regulated by clock genes. Briefly, Clock/Bmal1 induces Per1/2/3, Cry $1 / 2$, and differentiated embryonic chondrocyte gene $1 / 2$ (Decl/2) transcription through E-box. ${ }^{1-3}$ The complex of Period (Per) and Cryptochrome (Cry) protein then represses their own transcription by inhibiting transcriptional activity of Clock/ Bmal1. However, Dec suppresses their own transcription by competing with Clock/Bmal1 for the occupancy of the E-box in their own promoters. ${ }^{1-3}$ This negative feedback regulation by clock genes plays an important role in regulation of circadian rhythm. Dec1 and Dec2 are basic helix-loop-helix transcription factors, are ubiquitously expressed in whole tissues, and show circadian expression in the suprachiasmatic nucleus (SCN), peripheral tissues, mesenchymal stem cells, and tumor cells. ${ }^{3-9}$ Dec1 and Dec2 are induced by
Clock/Bmal1, hypoxia, serum shock, transforming growth factor (TGF)- $\beta$, and antitumor drugs, and they regulate circadian rhythm, sleep length, inflammation, lipid metabolism, adipogenic differentiation, the cell cycle, and tumor progression. ${ }^{5-10}$ Perl knockdown increased tumor proliferation and size in oral cancer cells, decreasing Dec1 and Dec2 expression. ${ }^{11}$ It has also been reported that Per1 and Dec2 have tissue-specific interactions in circadian rhythm regulation. ${ }^{12}$ Synergistic disruption of circadian rhythm in Perl and Dec1 or Dec2 double-mutant mice was observed in

Supported by JSPS KAKENHI grants 16K09624 (F.S.) and 15K08430 (Y.M.), and Nihon University Multidisciplinary Research Grant for 2018 (U.K.B.).

Disclosures: None declared. 
comparison with that in single-mutant mice. ${ }^{13}$ It seems that Dec1 or Dec2 interacts with Per1 in not only the regulation of circadian rhythm but also in various other phenomena.

Smad3 has circadian expression in the gingiva, liver, heart, and mesenchymal stem cells. ${ }^{7}{ }^{14} \mathrm{Smad} 3$ is induced by Clock/ Bmal1, serum shock, and TGF- $\beta$ and regulates cell proliferation, tumor progression, cardiac diseases, fibrosis, diabetes, and metabolic syndrome. ${ }^{7,15-18}$ The loss of Smad3 in mice promotes re-epithelialization and the restoration of connective tissue, resulting in faster wound healing than in wild-type mice. ${ }^{19-23}$ This result correlates with the alternation of wound healing-associated genes, decreased production of collagen I and fibronectin, and increased expression of $p 21$, S100A4, and PCNA. ${ }^{14,21,23-25}$ Several reports have shown that Smad3 affects Dec1 and Dec2 expression. ${ }^{17,26,27}$ For example, activation of TGF- $\beta$ signaling resets the circadian clock via Dec1. ${ }^{27}$ TGF- $\beta$ induced Smad3 phosphorylation and then induced Dec2 expression through Smad3-binding elements of the Dec2 promoter in pancreatic cancer cells. ${ }^{17,26}$ However, it is not yet clear how the loss of Smad3 affects circadian rhythm and Decl, Dec2, and Perl expression. Here, we investigated whether $\operatorname{Smad}^{-/-}(\operatorname{Smad} 3$ knockout) affects Decl, Dec2, and Perl expression in the $\mathrm{SCN}$ and peripheral tissues and how these genes interact in epithelial cell migration and proliferation.

\section{Materials and Methods}

\section{Animal and Tissue Preparation}

All animal experiments were performed according to protocols approved by the Animal Care and Use Committee of the Wakayama Medical University (approval number: 660). Six- to 8-week-old male and female $\operatorname{Smad} 3(+/+)$ wild-type and (-/-) whole knockout mice with a C57BL/ $6 \times 129$ SVEV background were generated as previously described. ${ }^{28}$ Mice were housed, and tissues were prepared as previously described. ${ }^{6,14,29}$ Forty $\mathrm{Smad}^{+/+}$and 40 $S m a d 3^{-1-}$ mice were used in this study. All mice were reared in 12:12 light/dark cycles (lights on at $8 \mathrm{AM}$, off at 8 $\mathrm{PM})$. With the use of isoflurane anesthesia, mice were sacrificed by cervical dislocation. A dim red light was used to sacrifice at Zeitgeber time (ZT)12 and ZT18. Mouse brain dissection was performed at $0.5 \mathrm{~mm}$ thickness, using a mouse brain coronal matrix (BrainScience Idea, Osaka, Japan), and the SCN and prefrontal cortex from the mice were punched out onto a chilled dish with the use of a 0.5$\mathrm{mm}$ diameter needle. The SCN and prefrontal cortex were quickly assessed for mRNA and protein purification.

\section{Activity Recordings}

Mouse locomotor activity was monitored by Supermex (Muromachi Kikai, Tokyo, Japan). Starting at 6 weeks of age, activity tests were conducted for 30 days. A sensor counts the movements of the mice by detecting the radiated body heat. The data were recorded continuously in 1-minute bins by a data collection program (CompACT AMS; Muromachi Kikai). The free-running period of locomotor activity was calculated by periodogram analysis with the use of ClockLab software (Actimetrics, Wilmette, IL). Fast Fourier analysis was performed to determine the amplitude of the power spectral density of the circadian peak.

\section{Wound-Healing Experiments of Mice}

Mice were anesthetized by injection of ketamine $(100 \mathrm{mg} / \mathrm{kg}$ of body weight) and xylazine $(5 \mathrm{mg} / \mathrm{kg}$ of body weight) in sterile phosphate-buffered saline, and the wound was made by punching out a $3.0-\mathrm{mm}$ hole with a Harris Uni-core puncher (BrainScience Idea) at the apex of the tongue. The mice were sacrificed at ZT6, and the tongue was prepared at 3 and 5 days after wounding. Tissues were fixed in $4 \%$ paraformaldehyde in phosphate-buffered saline (Wako, Osaka, Japan) and subjected to immunohistochemistry and hematoxylin and eosin staining.

\section{Real-Time PCR}

Three independent RNA samples were prepared from the mouse SCN, prefrontal cortex, and tongue. In addition, RNA was prepared from Smad3 overexpressing NIH3T3 cells. Total RNA was isolated and first-strand cDNA was synthesized as previously described. ${ }^{6,14}$ Real-time PCR was performed with the SYBR Green Master Mix (Bio-Rad Laboratories, Inc., Hercules, CA). The amplification primer sequences were designed as follows: Decl forward (F), 5'-ATCAGCCTCCTTTTTGCCTTC-3', and reverse (R), 5'-AGCATTTCTCCAGCATAGGCAG-3'; Dec2 F, 5'-ATTGCTTTACAGAATGGGGAGCG-3', and R, 5'-AAAGCGCGCGAGGTATTGCAAGAC-3'; Perl F, 5'-CCAGGATGTGGGTGTCTTCT-3,' and R, $5^{\prime}$-GTCCTTGAGACCTGAACCTG- $3^{\prime}$; and $18 S$ rRNA F, 5'-GCGCCGCTAGAGGTGAAAT-3', and R, 5'-GAAAACATTCTTGGCAAATGCTT- $3^{\prime}$. The data were normalized by using $18 S$ rRNA.

\section{Immunohistochemistry}

Dec1, Dec2, and Per1 expression in mouse kidney, liver, and tongue tissues was evaluated by using serial deparaffinized sections. Six $\operatorname{Smad} 3^{+/+}$and six $\operatorname{Smad} 3^{-/-}$mice were used for immunohistochemistry. Five serial sections of $4 \mu \mathrm{m}$ per organ were prepared for staining. Immunohistochemistry was performed by using a Discovery Auto-Stainer with automated protocols (Ventana Medical Systems, Inc., Tucson, AZ; Roche, Mannheim, Germany) as previously described. ${ }^{14}$

\section{Cell Culture and Treatment}

NIH3T3 mouse fibroblasts were obtained from ATCC (Manassas, VA). CA9-22 human gingival cancer cells were 
obtained from the Japanese Cancer Research Resources Bank (Osaka, Japan). These cells were cultured in Dulbecco's modified Eagle's medium (Sigma Chemical Co., St. Louis, MO) supplemented with $10 \%$ fetal bovine serum and $1 \%$ antibiotics. The construct expression vectors for human Dec1 and Dec2 were used for transfection. ${ }^{1}$ Human Smad3 plasmid (11742) was purchased from Addgene (Cambridge, MA). Transient plasmid transfection and siRNA transfection were performed as previously described. ${ }^{30}$ For Dec1 overexpressed-stable cells, empty (CA9-22 ${ }^{\text {vector }}$ ) and Dec1 (CA9-22 ${ }^{\text {Dec1 }}$ ) plasmids were transfected in cells. After transfection, medium was changed, including $1000 \mu \mathrm{g} / \mathrm{mL}$ G418 (Roche) solution every 3 days until 3 weeks and selected stable cells. The Dec1 induction was confirmed in the stable cells by Western blot analyses. Smad 3 siRNA was synthesized by Qiagen (Hilden, Germany). The sense and antisense siRNA sequences were as follows: Smad3 siRNA1, 5'-r (CAAGGGAUUUCCUAUGGAATT)- $3^{\prime}$ and $5^{\prime}$-r (UUCCAUAGGAAAUCCCUUGAT)- $3^{\prime} ; \quad \operatorname{Smad} 3$ siRNA2, $5^{\prime}$-r (GAGAUUCGAAUGACGGUAATT)- $3^{\prime}$ and $5^{\prime}$-r (UUACCGUCAUUCGAAUCUCTT)-3', respectively.

\section{Cell Migration Assay}

CA9-22 cells were seeded in a 12-well plate and transfected with control or Smad3 siRNA2. After 8 hours, the cells were transfected with empty, Dec1, or Dec2 plasmid, and then an artificial wound was carefully created at 0 hour by scratching the surface with the tip of a P-200 pipette. Pictures were taken by microscopic digital camera (Nikon DS-L3; Nikon Corporation, Tokyo, Japan) at 0 and 12 hours.

\section{Cell Proliferation Assay}

CA9-22 cells were seeded in 96-well plates. Transfection was performed as previously described. ${ }^{30}$ After 10 hours of transfection, the cells were added to each well by using a cell proliferation kit (XTT based) (Biological Industries, Kibbutz Beit Haemek, Israel) and incubated at $37^{\circ} \mathrm{C}$ for an additional 2 and 14 hours. The absorbance at $\mathrm{OD}_{480}$ and at $\mathrm{OD}_{650}$ was measured with the use of a 96-well microplate reader (SH-9000; Hitachi, Tokyo, Japan).

\section{Western Blot Analysis}

Individual mouse liver and kidney were lyzed with the use of T-PER lysis buffer (ThermoFisher Scientific, Yokohama, Japan). Three independent SCN samples were pooled and then were lyzed with the use of T-PER lysis buffer. Cells were lyzed with M-PER lysis buffer (ThermoFisher Scientific, Yokohama, Japan). Lysates were run on SDS-polyacrylamide gels followed by Western blot analysis with the use of standard procedures. A WesternBright Sirius Kit (Advansta, CA) was used for antibody detection, and an AE-9300 Ez capture MG (ATTO, Tokyo, Japan) was used for image data capture. The intensity of the bands detected on the Western blot analysis was quantified with ImageJ software version $1.47 \mathrm{v}(\mathrm{NIH}$, Bethesda, MD).

\section{Antibodies}

The following commercial antibodies were purchased: Smad3 (dilution 1:1000; rabbit monoclonal; ab40854; Abcam, Cambridge, UK), Dec1 (dilution 1:5000; rabbit polyclonal; NB100-1800; Novus Biologicals, Centennial, CO), Dec2 (dilution 1:2000; mouse monoclonal; sc-373763; Santa Cruz Biotechnology Inc., Santa Cruz, CA), proliferating cell nuclear antigen (PCNA; dilution 1:10,000; rabbit polyclonal; sc-7907; Santa Cruz Biotechnology Inc.), Per1 (dilution 1:1000; rabbit polyclonal; TransGenic Inc., Hyogo, Japan), E-cadherin (dilution 1:20,000; mouse monoclonal; M106; TaKaRa Bio Inc., Otsu, Japan), and actin (dilution 1:10,000; mouse monoclonal; A5441; Sigma Chemical Co.).

\section{Results}

\section{Smad3 Deficiency Decreases the Amplitude of Locomotor Activity}

It was first examined whether Smad3 deficiency affected circadian rhythm with the use of $S m a d 3^{-/-}$mice. Smad3 ${ }^{+/+}$ and $S m a d 3^{-1-}$ mice were fed under light/dark conditions for 14 days and constant dark conditions for 16 days. Locomotor activities were analyzed by Supermex. The amplitude of locomotor activity significantly decreased in Smad3 $3^{-/}$ mice compared with that in $\mathrm{Smad}^{+/+}$mice (Figure 1A). However, no significant differences were observed in the circadian period between $\mathrm{Smad}^{+/+}$and $\mathrm{Smad}^{-/-}$mice. Dec1, Dec2, and Perl mRNA expression was next examined at ZT6 and ZT12 in the SCN of $\mathrm{Smad3}^{+/+}$and $\mathrm{Smad}^{-/-}$mice by real-time PCR, because time difference may affect clock gene expression. Decl expression was higher, whereas Dec2 and Perl expression was lower at both time points in $\mathrm{Smad}^{+/+}$mice than in $\mathrm{Smad}^{-/-}$mice (Figure 1, B and C), suggesting that Smad3 up-regulates Decl expression and down-regulates Dec2 and Perl expression. In addition, Dec1, Dec2, and Per1 protein expression was analyzed at ZT6 and ZT12 in the SCN, and the result was similar at mRNA levels (Figure 1D). Decl, $D e c 2$, and Perl expression was found to be higher in the $\mathrm{SCN}$ of $\mathrm{Smad}^{+/+}$mice than in the prefrontal cortex (Supplemental Figure S1).

Diurnal Variation in the Expression of Dec1, Dec2, and Per1 in the Kidney

To examine Dec1, Dec2, and Per1 protein expression in $\mathrm{Smad}^{+/+}$and $\mathrm{Smad}^{-/-}$mice, Western blot analysis was performed. Compared with that in $\mathrm{Smad}^{+/+}$mice, Dec1 protein expression at ZT6 decreased in the liver and kidneys of $\mathrm{Smad}^{-/-}$mice, whereas Dec2 and Per1 protein expression increased (Figure 2A). Because the protein levels 

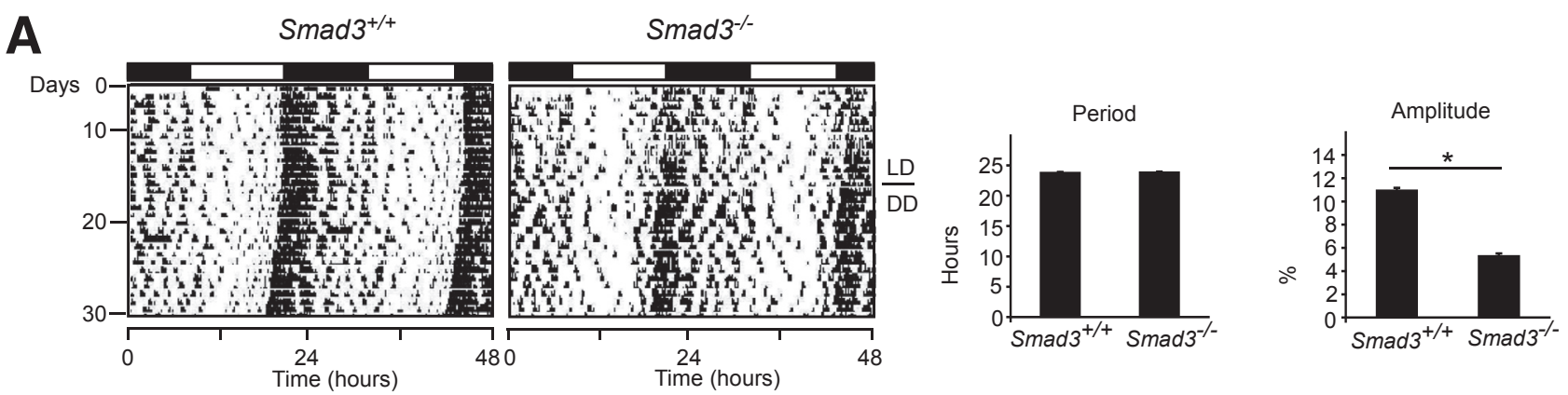

B

ZT6
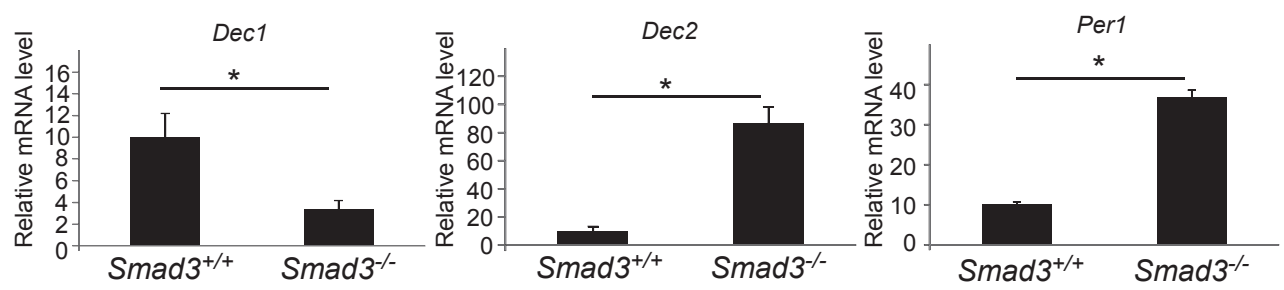

C
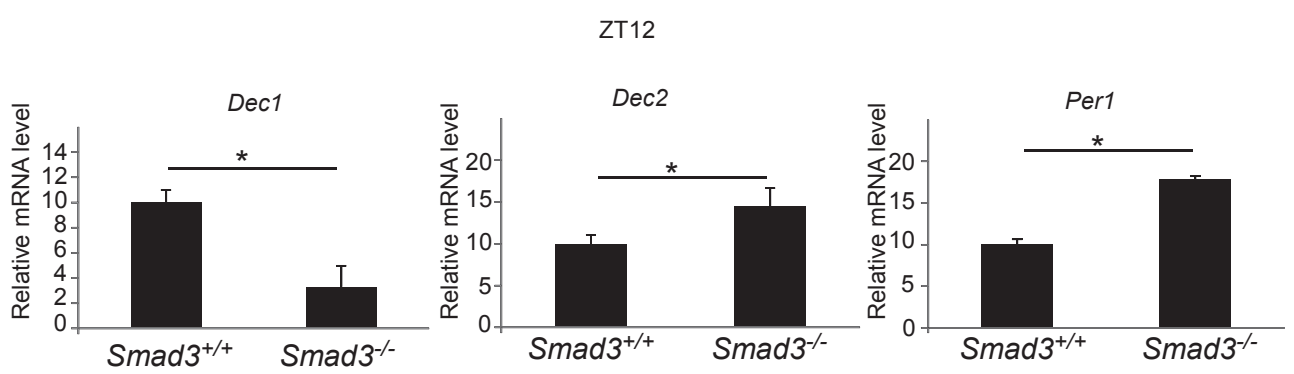

D

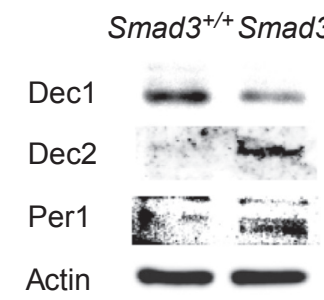

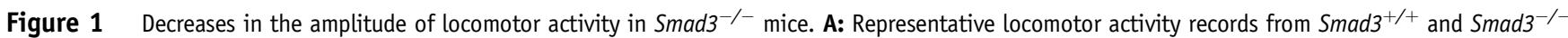
mice under 12:12 light/dark (LD; 14 days) and constant darkness (DD; 16 days) cycles. Black squares indicate the darkness. The records are double-plotted such that 48 hours are shown for each horizontal trace. The period and amplitude of Smad3 ${ }^{+/+}$and Smad $3^{-/-}$mice under DD are also shown. B and C: Relative Dec1, Dec2, and Per1 mRNA expression in the suprachiasmatic nucleus (SCN) of Smad $3^{+/+}$and Smad3 $3^{-/-}$mice at Zeitgeber time (ZT)6 (B) and ZT12 (C) was analyzed by real-time PCR. The data were normalized to $18 S$ rRNA. D: Differentiated embryonic chondrocyte (Dec)1, Dec2, period (Per)1, and actin protein expression in the SCN of Smad3 $3^{+/+}$and $\mathrm{Smad}^{-/-}$mice at ZT6 and ZT12. Data are expressed as means \pm SEM. $n=3$ independent samples. ${ }^{\star} P<0.05$ ( $t$-test).

of Dec2 was virtually undetectable in the liver of $\mathrm{Smad}^{+/+}$, the circadian expression of Dec2 and other clock genes was examined specifically in the kidneys of $\operatorname{Smad}^{+/+}$and $\mathrm{Smad}^{-/-}$mice. Circadian expression of Smad3 in $\mathrm{Smad}^{+/+}$ mice was also examined. As expected, Dec1, Dec2, Per1, and Smad3 protein expression showed diurnal variation in $\mathrm{Smad3}^{+/+}$mice (Figure 2B). Smad3 ${ }^{-/-}$mice also showed diurnal variation in the protein expression of these circadian genes in the kidneys; however, the expression levels were altered. Specifically, Dec1 protein expression decreased from ZT0 to ZT18 in the kidneys of Smad $3^{-/-}$mice compared with that in $\mathrm{Smad}^{+/+}$mice. Interestingly, Dec2 protein expression greatly increased from ZT0 to ZT18 in the kidneys of $S m a d 3^{-1}$ - mice compared with that in $S m a d 3^{+/+}$mice. Per1 protein expression was slightly increased in the kidneys of $\operatorname{Smad} 3^{-1-}$ mice compared with $\mathrm{Smad}^{+/+}$mice. 
A

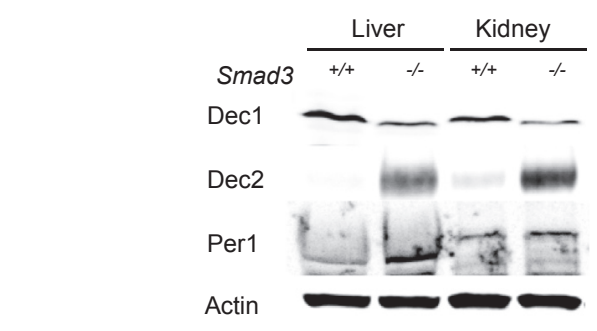

B
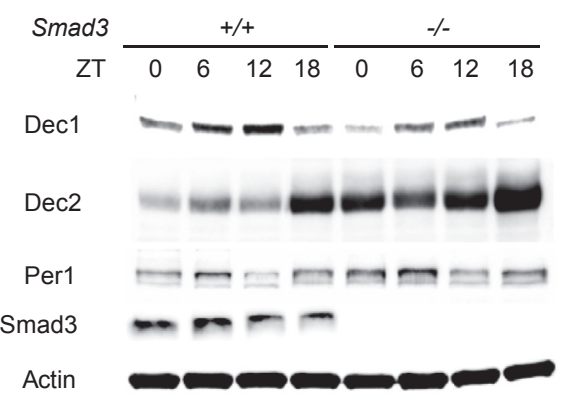

Dec1
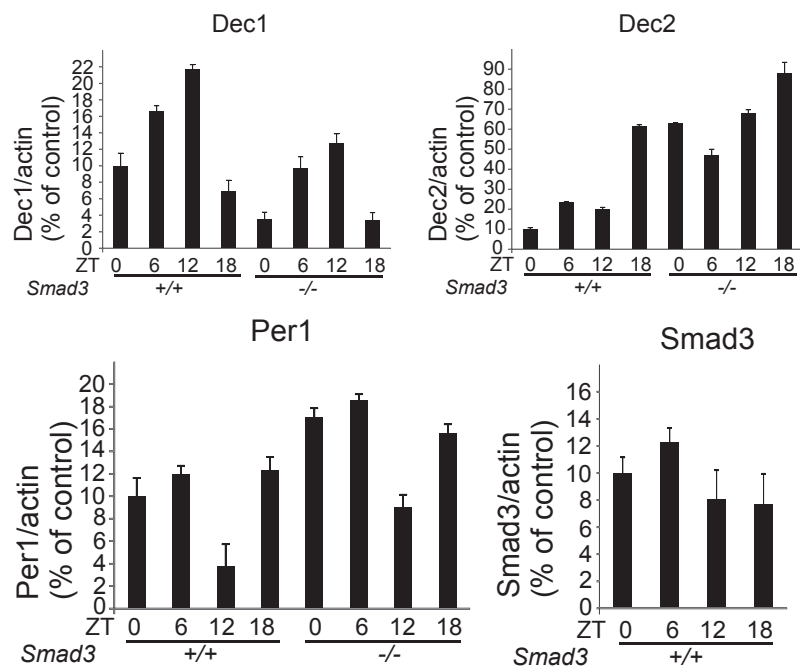

Figure 2 Diurnal variation in differentiated embryonic chondrocyte (Dec)1, Dec2, and period (Per)1 expression in Smad3 $3^{-/-}$mouse livers and kidneys. A: Representative Western blot analyses of Dec1, Dec2, Per1, and actin at Zeitgeber time (ZT) 6 in the liver and kidneys of $\mathrm{Smad}^{+/+}$and Smad3 ${ }^{-/-}$mice. B: Representative Western blot analyses of Dec1, Dec2, and Per1 circadian expression in the kidneys of $\mathrm{Smad3}^{+/+}$and $\mathrm{Smad3}^{-/-}$ mice (top panel). Actin was used as a loading control. Quantitation of the intensities of bands in the data from Western blot analysis (bottom panels). The data were normalized to actin. Data are expressed as means \pm SEM. $n=3$ independent samples.

\section{Smad3 Deficiency Down-Regulates Dec1 Expression,} Up-Regulating Dec2 and Per1 Expression in Endothelial Cells of Mouse Kidney and Liver

To understand the localization and expression of Dec1, Dec2, and Per1, immunohistochemistry was performed with the use of kidney and liver tissues of $\operatorname{Smad}^{+/+}$and Smad3 ${ }^{-1-}$ mice. Strong positive staining for Dec1 was detected in endothelial cells in the kidney and liver and in renal tubular epithelial cells of $\mathrm{Smad}^{+/+}$mice, whereas no significant immunostaining was observed in those of Smad3 ${ }^{-/-}$mice (Figure 3A). In contrast, strong positive staining for Dec2 and Per1 was detected in endothelial cells in the kidneys and liver of $\operatorname{Smad3}^{-/-}$mice, but not in those of $\mathrm{Smad3}^{+/+}$mice (Figure 3, B and C). These results suggested that Smad3 regulated Dec1 expression and negatively regulated Dec2 and Per1 expression.

\section{Smad3 Overexpression Increases Dec1 Expression, Decreasing Dec2 and Per1 Expression}

Next, it was examined whether overexpression by Smad3 plasmid affected Dec1, Dec2, and Per1 expression with the use of NIH3T3 cells. Smad3 overexpression increased Dec1 protein expression in NIH3T3 cells compared with control treated cells (Figure 4A). Endogenous protein expression of Dec2 and Per1 was low in NIH3T3 cells. Therefore, realtime PCR was performed to assess the mRNA levels. Smad3 overexpression increased Decl mRNA expression but decreased Dec2 and Perl mRNA expression (Figure 4B). These results were in good agreement with immunohistochemistry in mouse tissues.

\section{Wound Healing Is Promoted by the Alternation of Clock Gene Expression in Smad3 ${ }^{-/-}$Mice}

It was further examined how Dec1, Dec2, and Per1 expression was affected by wound healing in $S$ mad $3^{+/+}$and Smad3 ${ }^{-/-}$mice. On day 3 and day 5 after wounding, reepithelialization of the tongue was accelerated in $\mathrm{Smad}^{-/-}$mice compared with $\mathrm{Smad}^{+/+}$mice, whereas the degree of inflammation decreased in $\operatorname{Smad3}^{-/-}$mice (Supplemental Figure S2). On day 3 ZT6 after wounding, positive staining for Dec1 was dominantly observed in basal cells of the adjacent squamous epithelium of $\mathrm{Smad}^{+/+}$mice, whereas positively stained cells were significantly decreased in the wound lesions (Figure 5A). By contrast, the intensity of immunostaining for Dec1 was weak in both the adjacent squamous epithelium and wound lesions of Smad3 ${ }^{-/-}$mice. However, positive staining for PCNA, Dec2, and Per1 was weak in basal cells of the adjacent squamous epithelium of $\mathrm{Smad}^{+/+}$mice, whereas a number of cells positive for PCNA, Dec2, and Per1 expression were observed in $S_{m a d}{ }^{-/-}$mice (Figure $5 \mathrm{~A}$ and Supplemental Figure S3). Positive staining between Dec1 and PCNA had a significant correlation. In addition, Decl mRNA expression was decreased in $\mathrm{Smad3}^{-1-}$ mice compared with that in $\mathrm{Smad}^{+/+}$mice (Figure 5B). On the fifth day after wounding, a similar immunostaining pattern for Dec1, Dec2, and Per1 was observed in $\operatorname{Smad}^{+/+}$and $\mathrm{Smad3}^{-/-}$mice (Supplemental Figure S4). From the above data, it was hypothesized that Smad3 suppressed basal cell 
A
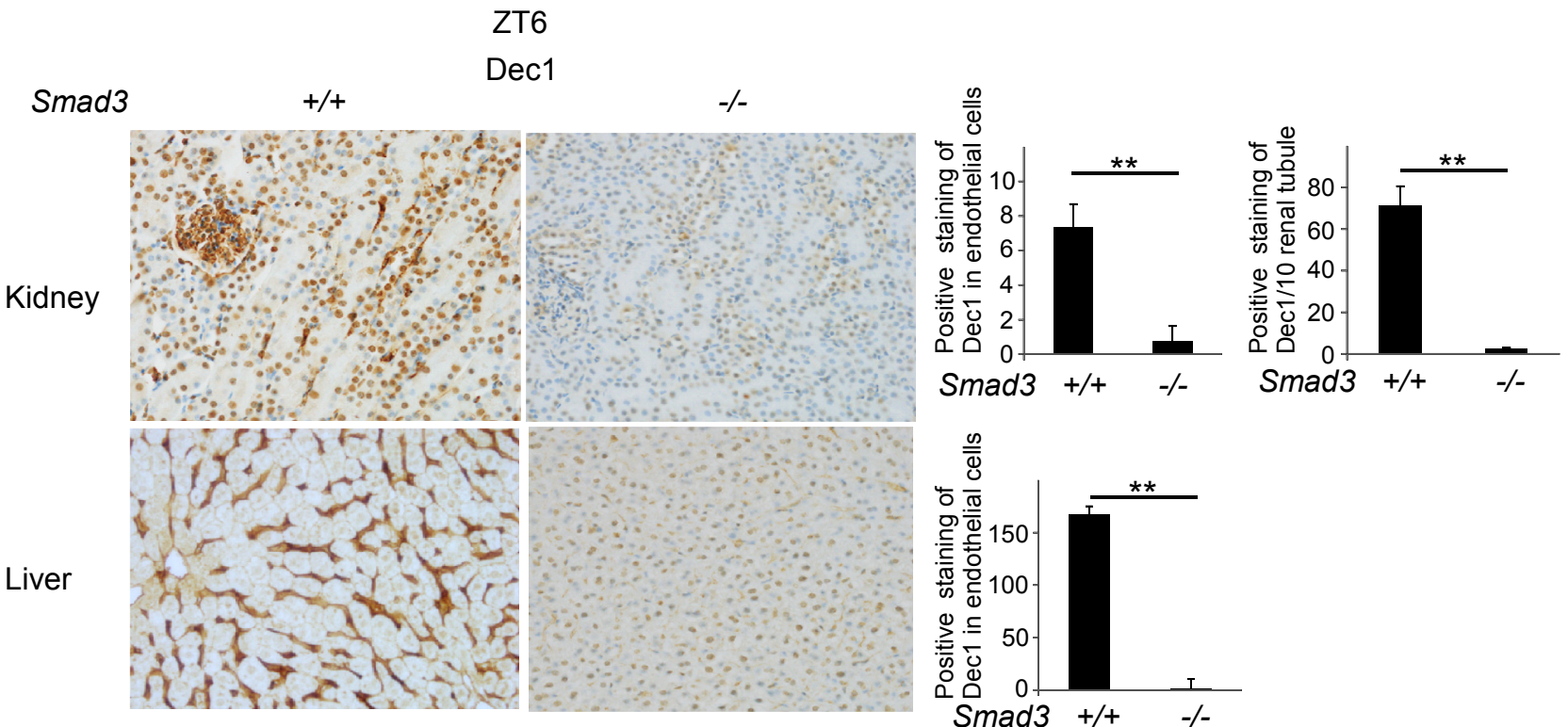

B
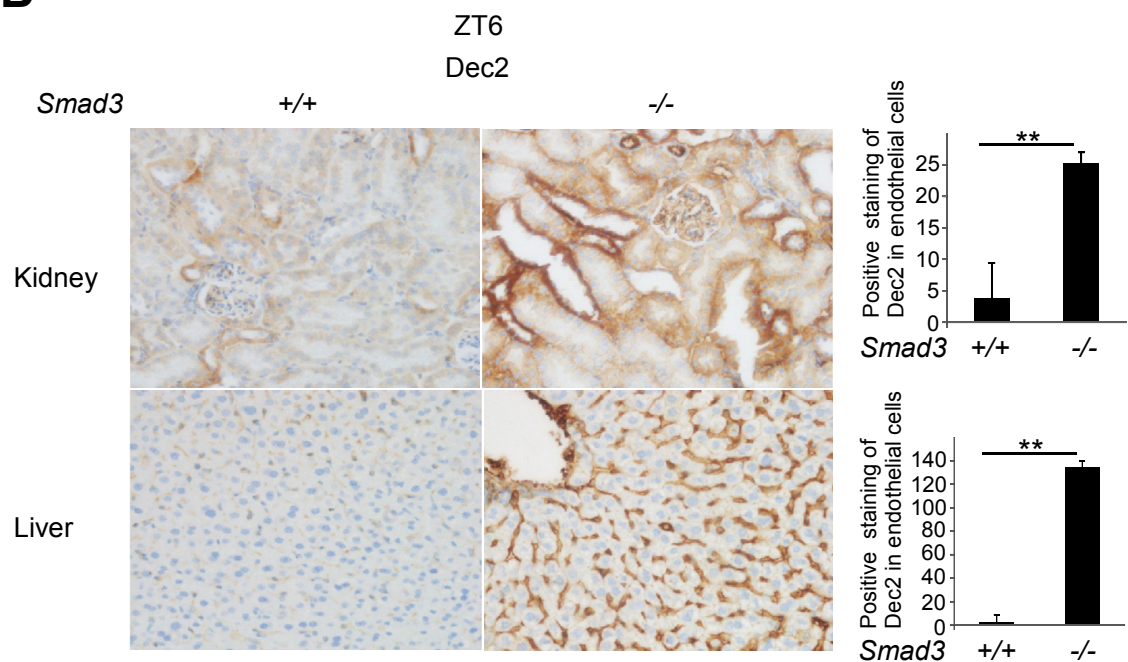

Figure 3 Immunohistochemical detection of differentiated embryonic chondrocyte (Dec)1, Dec2, and period (Per)1 in the kidneys and liver of $\mathrm{Smad3}^{+/+}$and

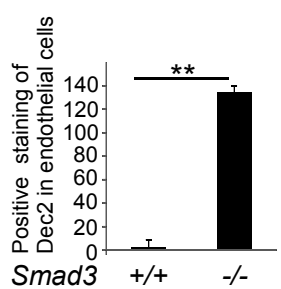

C

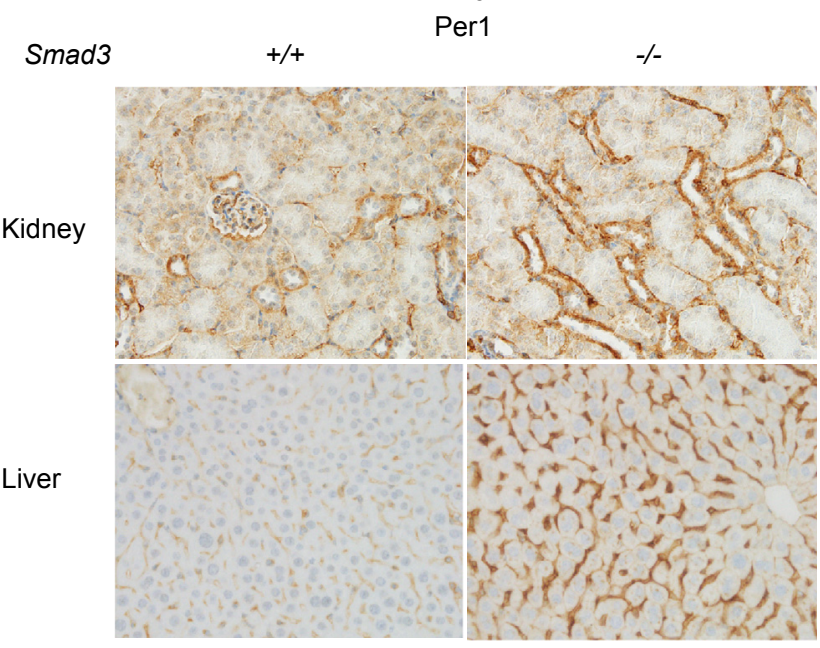

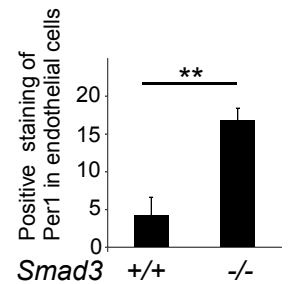

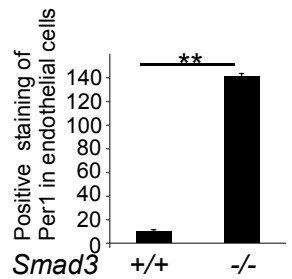
Smad3 $^{-/-}$mice. A-C: Representative images for immunohistochemistry of Dec1 (A), Dec2 (B), and Per1 (C) at Zeitgeber time (ZT)6 in the kidneys and liver of $\mathrm{Smad}^{+/+}$and $\mathrm{Smad}^{-1-}$ mice (left panel). Positive staining for Dec1, Dec2, and Per1 in endothelial cells of Smad3 $3^{+/+}$ and $\mathrm{Smad3}^{-/-}$mouse kidneys and liver was counted in nine random microscopic fields (right panel). In addition, positive staining for Dec1 in 10 renal tubules was counted as above. Data are expressed as means \pm SEM. $n=3$ independent samples. ${ }^{* *} P<0.01$ (t-test). Original magnification, $\times 400(\mathrm{~A}-\mathrm{C})$. 
A

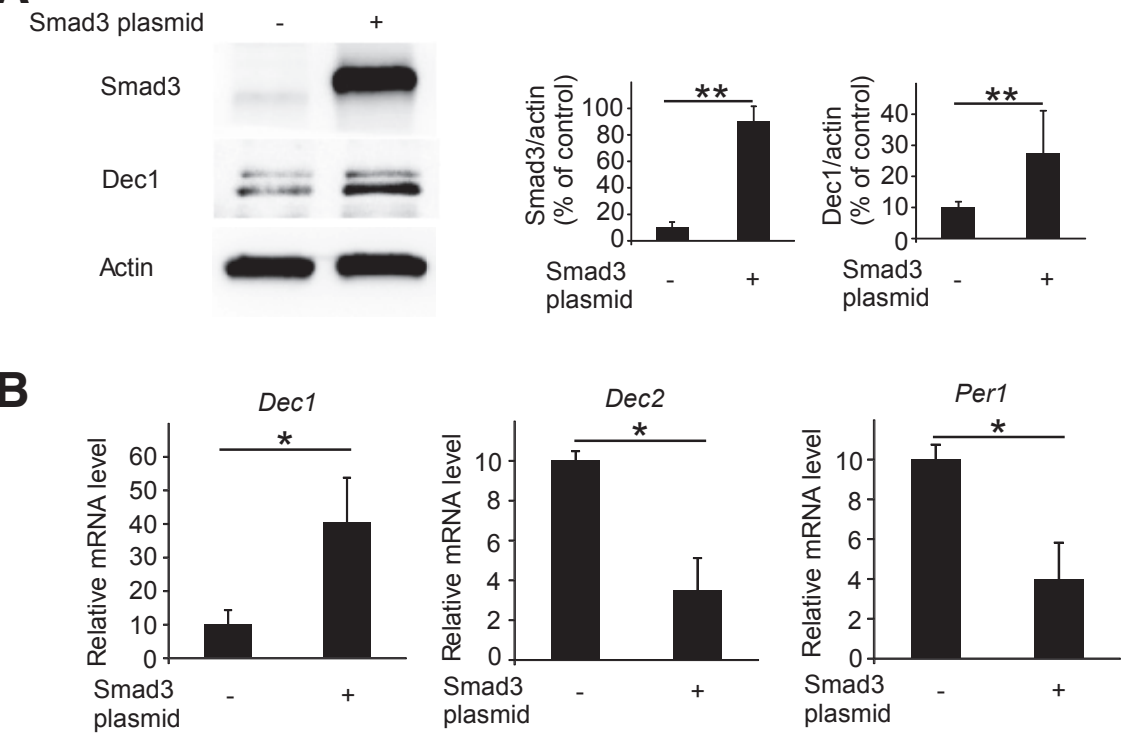

Figure 4 Smad3 overexpression increases differentiated embryonic chondrocyte (Dec)1 expression but decreases Dec2 and period (Per)1 expression. A: Representative Western blot analyses of Smad3, Dec1, and actin expression in NIH3T3 cells in the presence $(+)$ or absence $(-)$ of Smad3 plasmid (left panel). Quantitation of the intensities of bands in the data from the Western blot analysis (right panels). B: Relative Dec1, Dec2, and Per1 mRNA expression in the presence or absence of Smad3 plasmid from NIH3T3 cells as measured by real-time PCR. The data were normalized to 185 rRNA. Data are expressed as means \pm SEM. $n=3$ independent samples. ${ }^{*} P<0.05$ and ${ }^{* *} P<0.01$ ( $t$-test).

proliferation in re-epithelialization during wound healing via Dec1, which in turn down-regulated Dec2 and Per1 expression.

\section{Smad3 Regulates Cell Migration and Proliferation via Dec1 and Dec2}

To explore this hypothesis, it was examined whether Smad3 knockdown with or without Dec1 or Dec2 overexpression affected the migration and proliferation of human oral squamous cancer CA9-22 cells. Both siRNA1 and siRNA2 for $S m a d 3$ knockdown greatly decreased endogenous Smad3 expression in CA9-22 cells (Figure 6A). Smad3 siRNA2 had effective knockdown compared with Smad3 siRNA1. Dec1 overexpression greatly increased exogenous Dec1 expression, but it decreased endogenous Dec2, Per1, E-cadherin, and PCNA expression in CA9-22 cells (Figure 6A). However, Dec2 overexpression increased both E-cadherin and PCNA expression, whereas it had little effect on Dec1 and Per1 (Figure 6A). After a wound was created at 12 hours, Smad3 knockdown by siRNA2 greatly induced cell migration compared with control siRNA (Figure 6B). However, Dec1 overexpression with or without Smad3 siRNA2 greatly suppressed cell migration. In addition, Dec1 overexpression with or without Smad3 siRNA2 significantly decreased cell proliferation (Figure 6C). Migration and cell proliferation assays were also performed with the use of Dec1 overexpressed-stable cells. The results were compatible with transient transfection experiments (Supplemental Figure S5). Conversely, Dec2 overexpression with or without Smad3 siRNA2 significantly induced cell migration and proliferation. These results suggested that cell migration and proliferation were promoted by Smad3 deficiency through Dec1 down-regulation and Dec2 up-regulation.

\section{Discussion}

The decreases in the circadian amplitude imply the disruption of activity patterns and sleep quality. In addition, these decreases are associated with metabolic syndrome, depression, and cardiac diseases. ${ }^{31,32}$ Because $S m a d 3^{-/-}$mice experienced great decreases in the amplitude of locomotor activity, it was hypothesized that it may depend on the abnormality of clock genes through Smad3 deficiency. As expected, alternation of Decl, Dec2, and Perl expression was observed in the SCN, liver, kidneys, and tongue in $\operatorname{Smad}^{+/+}$and $\operatorname{Smad}^{-/-}$ mice. In addition, Bmall expression decreases in the heart of $\mathrm{Smad}^{-/-}$mice compared with that in control mice. ${ }^{14}$ This finding implies that $\operatorname{Smad} 3$ deficiency certainly alters the expression of clock genes. Meanwhile, Smad3 $3^{-1-}$ mice did not show significant change during the circadian period. Dec1, Dec2, and Per1 had circadian expression in the SCN and kidneys of Smad $3^{-/-}$mice, so the circadian period may have a minimal effect. Smad3 may compensate for other Smads, such as Smad2. In other studies, Decl or Perl single-knockout mice had only a slight change in circadian rhythm, whereas Decl/Dec2 or Perl/ Per2 double-knockout mice had a marked change. ${ }^{33,34}$ Similarly, Smad3 forms a heterodimer with Smad2 and is bound to the DNA of target genes. ${ }^{35}$ Smad2, therefore, may compensate for Smad3 deficiency. Smad $3^{-1}$ mice have a circadian rhythm in central and peripheral tissues, although the amplitude of the locomotor activity and the expression of Dec1 and Dec2 are dampened. Note that Decl and Dec2 double mutant mice reduce the amplitude of sleep-wake cycle. ${ }^{33}$ Therefore, the dampened amplitude of activity rhythm in $\operatorname{Smad}^{-1-}$ mice may be induced by the altered expressions of both Decl and Dec2 observed in the SCN. 
A

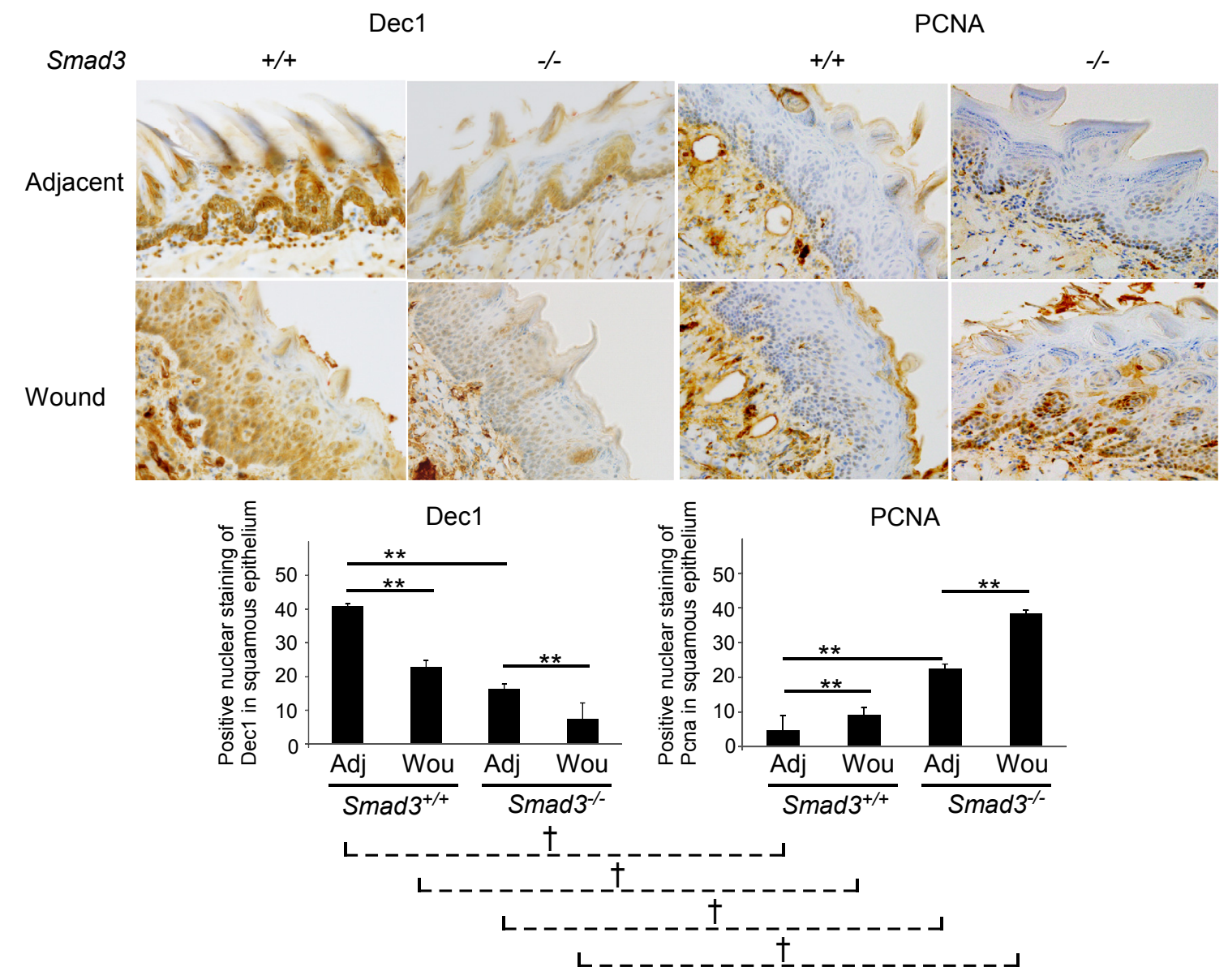

B

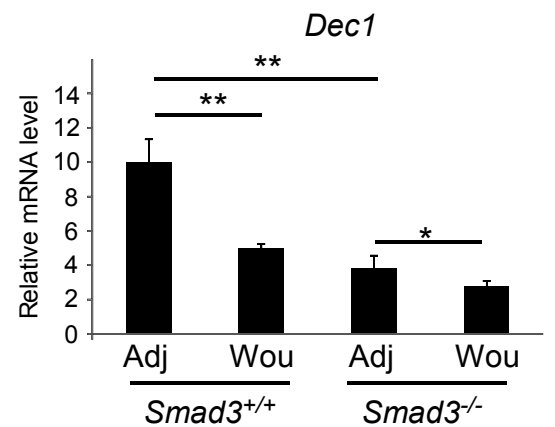

Figure 5 Differentiated embryonic chondrocyte (Dec)1 expression decreased in basal cells of the squamous epithelium of the tongue of $\mathrm{Smad3}^{-/-}$mice. A: Representative images of immunohistochemistry for Dec1 and proliferating cell nuclear antigen (PCNA) at Zeitgeber time (ZT)6 in the tongue of $\mathrm{Smad3}^{+/+}$and $\mathrm{Smad3}^{-/-}$mice 3 days after punch biopsy (top panels). Positive nuclear staining for Dec1 and PCNA in the squamous epithelium was counted in nine random microscopic fields (bottom panels). Immunostaining data of Dec2 and Per1 were included in the Supplemental Figure S3. B: Relative Dec1 mRNA expression in the adjacent (Adj) and wound lesions (Wou) of $\mathrm{Smad3}^{+/+}$and $\mathrm{Smad}^{-/-}$mouse tongue at ZT6 was analyzed by real-time PCR. The data were normalized to $18 S$ rRNA. The average numbers are shown (A). Data are expressed as means \pm SEM. $n=3$ independent samples. ${ }^{* *} P<0.01$ [multiple comparison-Dunnett test (straight)]; ${ }^{\dagger} P<0.05$ (Bonferroni test); ${ }^{\ddagger} P<0.05$ (Dunnett test). Original magnification, $\times 400$.
There is accumulating evidence that Dec1, Dec2, and Per1 play important roles not only in circadian rhythm but also in apoptosis, differentiation, and disease progression. . $^{1,431,36-41}$ In other words, Dec1, Dec2, and Per1 exert their effects under stress. Several reports have shown that Dec1 overexpression affects the expression of clock genes in vitro. ${ }^{42,43}$ Nevertheless, the differential expression of Dec1, Dec2, and Per1 in vivo has been unclear. It has been proved that Smad3 knockout accelerates wound healing. ${ }^{20-23}$ As a stress, wounding was performed by punch biopsy, and it was investigated whether Smad3 deficiency affects Dec1, Dec2, and Per1 expression during wound healing. The tongue is quickly repaired after wounding, and punch biopsy of tongue causes comparatively little damage to the mouse compared with liver and kidney. Wound healing was therefore performed on the tongue. By wounding the tongue of Smad3 knockout mice, Dec1 expression decreased in the basal cells of the squamous epithelium, whereas Dec2 and Per1 expression increased. Although it is unclear how Dec2 and Per1 expression increased, a clear negative relationship was 
A

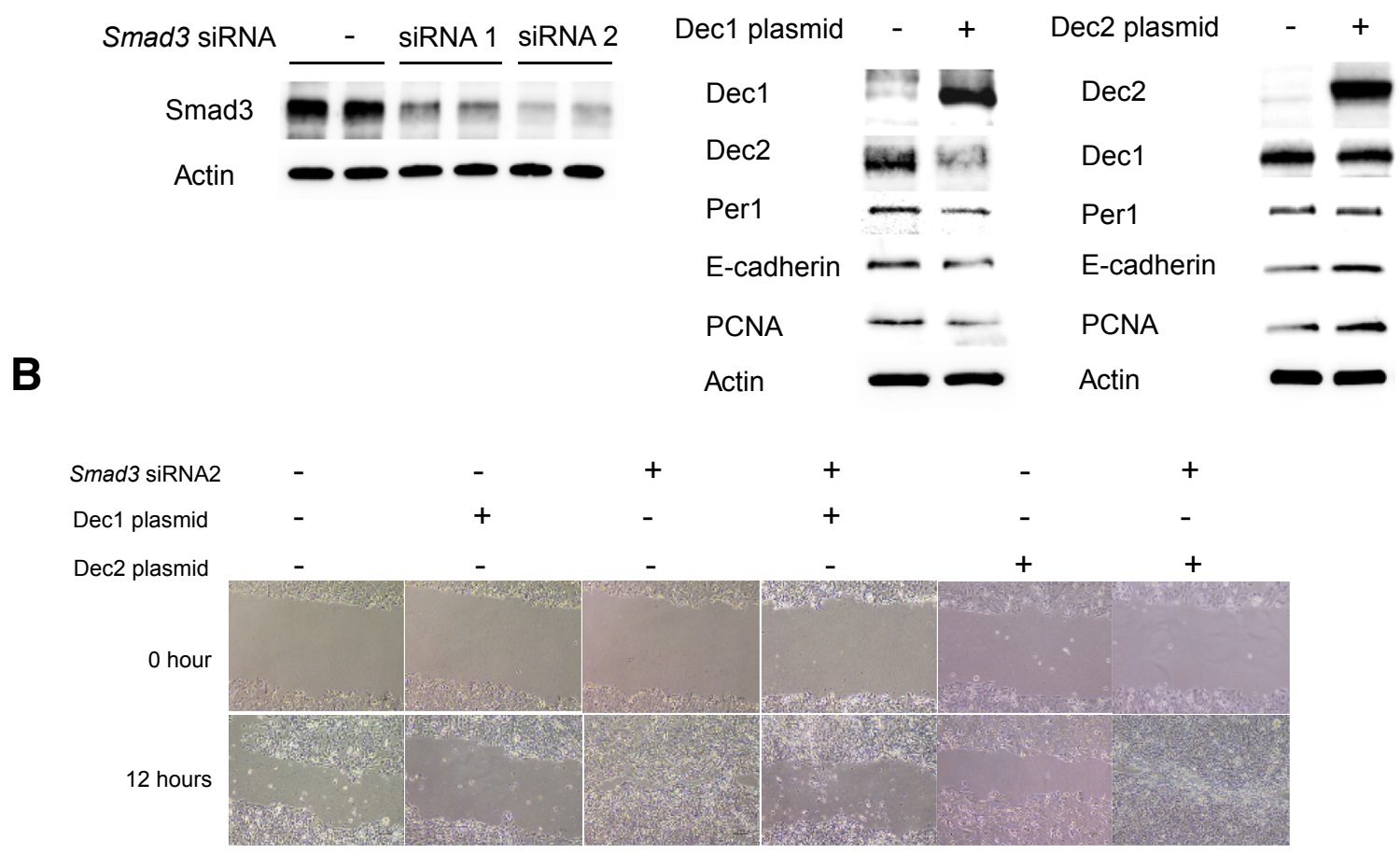

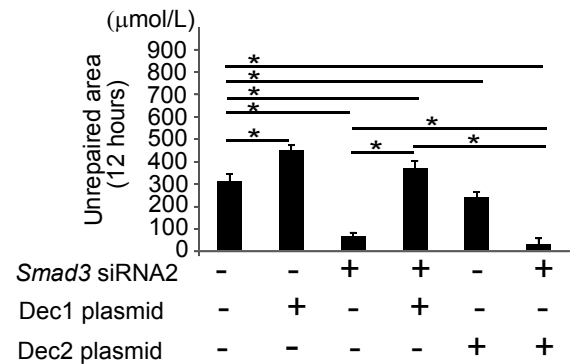

C

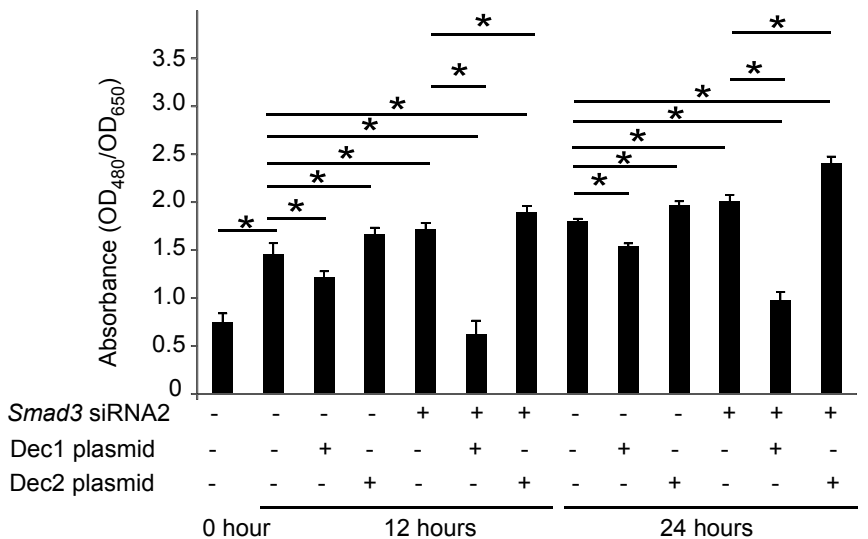

Figure 6 Differentiated embryonic chondrocyte (Dec)1 overexpression suppressed whereas Dec2 overexpression induced the migration and proliferation of oral squamous carcinoma cells. A: Representative Western blot analyses of Smad3 and actin by Smad3 siRNA1 or Smad3 siRNA2 in CA9-22 cells. The samples were prepared from two independent wells (left panel). Representative Western blot analyses of Dec1, Dec2, period (Per)1, E-cadherin, proliferating cell nuclear antigen (PCNA), and actin protein expression in Dec1- or Dec2-overexpressing CA9-22 cells (middle and right panels). B: Representative images for the cell migration assay (top panels). CA9-22 cells were transfected with control or Smad3 siRNA2. After 8 hours, the cells were transfected with control, Dec1, or Dec2 plasmid, and then a wound was made with the use of a pipette tip. Photographs of the wound area were taken at 0 and 12 hours. The three different unrepaired areas in each well were measured by scale and quantified (bottom panel). The average unrepaired areas at 12 hours are shown. C: Cell proliferation assay. CA9-22 cells were treated with $(+)$ or without $(-)$ Smad3 SiRNA2 and Dec1 or Dec2 plasmid. The absorbance $\left(0_{480} / 0_{650}\right)$ at 0 , 12 , and 24 hours is shown. Data are expressed as means \pm SEM. $n=3$ independent samples (B). ${ }^{*} P<0.05$ (Dunnett test). Original magnification, $\times 100$. found between Dec1 and Dec2 and Per1. In vitro experiments revealed that Dec1 overexpression suppressed cell migration and proliferation, whereas Dec2 overexpression had the opposite effect on the cells. This finding implies that the suppression of cell migration and proliferation by Smad3 may occur via Dec1. Moreover, these results demonstrated that Dec1 and Dec2 regulate cell migration and proliferation via E-cadherin and PCNA. In particular, Dec1 had significant 
correlation with PCNA in wound-healing experiments. In addition, Dec1 overexpression decreased PCNA expression in vitro. Dec1 function in the regulatory mechanisms of cell proliferation is not well understood. Notably, Dec1 suppressed proliferation, inhibiting PCNA.

The interaction between $\operatorname{Smad} 3$ and Dec2 and Per1 is likely as follows: First, Smad3 increases the Dec1 expression, although it decreases the Dec2 and Per1 expression. Second, although Dec1 overexpression decreases the Dec2 and Per1 expression, Dec2 overexpression has little effect on the Dec1 and Per1 expression. Third, Dec1 is induced early by stresses, whereas Dec2 is induced later. $3,4,7,32$ Collectively, these results suggest that Smad3 negatively regulates Dec2 and Per1 expressions via Dec1. Furthermore, in wound-healing experiments that used $\mathrm{Smad}^{+/+}$and Smad3 ${ }^{-/-}$mice, it was revealed that Dec1 expression was decreased in the wound lesions, even in $S m a d 3^{+/+}$mice. Tumor necrosis factor- $\alpha$, which is a suppressor of TGF- $\beta$, also suppresses Smad3 and Dec1 expression in NIH3T3 cells. ${ }^{14}$ It is possible that tumor necrosis factor- $\alpha$ production in wound lesions may influence Smad3 and Dec1 expression. Intriguingly, a recent study has demonstrated that the actin cytoskeleton of fibroblasts has rhythmic expression, influencing wound-healing efficacy. ${ }^{44}$ Smad3 expression may be involved in the mechanism by which tongue wounds incurred during daytime heal faster than nighttime wounds. Although it was found that the alternation in the healing processes observed under the Smad3-deficient condition is associated with Dec1 and Dec2 function, further studies are needed to elucidate the precise mechanisms of wound healing. These issues include the roles of Smad3 and clock genes in squamous epithelium and fibroblasts.

In summary, Smad3 deficiency alters Dec1, Dec2, and Perl expression in circadian central and peripheral tissues. We propose that Smad3 is a new regulator of clock genes that are involved in circadian rhythm, wound healing, and cell proliferation and migration. Investigation of the interaction between Smad3 and clock genes may shed light on chronotherapy alternatives for wound healing and cancer.

\section{Acknowledgments}

F.S., T.O., and H.T.L. performed experiments; A.K. and U.K.B. analyzed data; F.S. prepared the draft and made figures; A.K. and Y.M. edited the manuscript.

\section{Supplemental Data}

Supplemental material for this article can be found at https://doi.org/10.1016/j.ajpath.2019.01.006.

\section{References}

1. Sato F, Kawamoto T, Fujimoto K, Noshiro M, Honda KK, Honma S, Honma K, Kato Y: Functional analysis of the basic helix-loop-helix transcription factor DEC1 in circadian regulation. Interaction with BMAL1. Eur J Biochem 2004, 271:4409-4419

2. Kawamoto T, Noshiro M, Sato F, Maemura K, Takeda N, Nagai R, Iwata T, Fujimoto K, Furukawa M, Miyazaki K, Honma S, Honma Ki, Kato Y: A novel autofeedback loop of Dec1 transcription involved in circadian rhythm regulation. Biochem Biophys Res Commun 2004, 313:117-124

3. Honma S, Kawamoto T, Takagi Y, Fujimoto K, Sato F, Noshiro M, Kato Y, Honma K: Dec1 and Dec2 are regulators of the mammalian molecular clock. Nature 2002, 419:841-844

4. Sato F, Bhawal UK, Yoshimura T, Muragaki Y: DEC1 and DEC2 crosstalk between circadian rhythm and tumor progression. J Cancer 2016, 7:153-159

5. Sato F, Muragaki Y, Kawamoto T, Fujimoto K, Kato Y, Zhang Y: Rhythmic expression of DEC2 protein in vitro and in vivo. Biomed Rep 2016, 4:704-710

6. Sato F, Sato H, Jin D, Bhawal UK, Wu Y, Noshiro M, Kawamoto T, Fujimoto K, Seino H, Morohashi S, Kato Y, Kijima H: Smad3 and Snail show circadian expression in human gingival fibroblasts, human mesenchymal stem cell, and in mouse liver. Biochem Biophys Res Commun 2012, 419:441-446

7. Sato F, Bhawal UK, Kawamoto T, Fujimoto K, Imaizumi T, Imanaka T, Kondo J, Koyanagi S, Noshiro M, Yoshida H, Kusumi T, Kato Y, Kijima H: Basic-helix-loop-helix (bHLH) transcription factor DEC2 negatively regulates vascular endothelial growth factor expression. Genes Cells 2008, 13:131-144

8. Fujimoto K, Shen M, Noshiro M, Matsubara K, Shingu S, Honda K, Yoshida E, Suardita K, Matsuda Y, Kato Y: Molecular cloning and characterization of DEC2, a new member of basic helix-loop-helix proteins. Biochem Biophys Res Commun 2001, 280:164-171

9. Shen M, Kawamoto T, Yan W, Nakamasu K, Tamagami M, Koyano Y, Noshiro M, Kato Y: Molecular characterization of the novel basic helix-loop-helix protein DEC1 expressed in differentiated human embryo chondrocytes. Biochem Biophys Res Commun 1997, 236:294-298

10. Fujita Y, Makishima M, Bhawal UK: Differentiated embryo chondrocyte 1 (DEC1) is a novel negative regulator of hepatic fibroblast growth factor 21 (FGF21) in aging mice. Biochem Biophys Res Commun 2016, 469:477-482

11. Zhao Q, Zheng G, Yang K, Ao YR, Su XL, Li Y, Lv XQ: The clock gene PER1 plays an important role in regulating the clock gene network in human oral squamous cell carcinoma cells. Oncotarget 2016, 7:70290-70302

12. Tsang AH, Sánchez-Moreno C, Bode B, Rossner MJ, Garaulet M, Oster H: Tissue-specific interaction of Per1/2 and Dec2 in the regulation of fibroblast circadian rhythms. J Biol Rhythms 2012, 27:478-489

13. Bode B, Shahmoradi A, Taneja R, Rossner MJ, Oster H: Genetic interaction of Per1 and Dec1/2 in the regulation of circadian locomotor activity. J Biol Rhythms 2011, 26:530-540

14. Sato F, Kohsaka A, Takahashi K, Otao S, Kitada Y, Iwasaki Y, Muragaki Y: Smad3 and Bmal1 regulate p21 and S100A4 expression in myocardial stromal fibroblasts via TNF- $\alpha$. Histochem Cell Biol 2017, 148:617-624

15. Soofi A, Wolf KI, Emont MP, Qi N, Martinez-Santibanez G, Grimley E, Ostwani W, Dressler GR: The kielin/chordin-like protein (KCP) attenuates high-fat diet-induced obesity and metabolic syndrome in mice. J Biol Chem 2017, 292:9051-9062

16. Biernacka A, Cavalera M, Wang J, Russo I, Shinde A, Kong P Gonzalez-Quesada C, Rai V, Dobaczewski M, Lee DW, Wang XF, Frangogiannis NG: Smad3 signaling promotes fibrosis while preserving cardiac and aortic geometry in obese diabetic mice. Circ Heart Fail 2015, 8:788-798

17. Wu Y, Sato F, Yamada T, Bhawal UK, Kawamoto T, Fujimoto K, Noshiro M, Seino H, Morohashi S, Hakamada K, Abiko Y, Kato Y, Kijima H: The BHLH transcription factor DEC1 plays an important role in the epithelial-mesenchymal transition of pancreatic cancer. Int $\mathrm{J}$ Oncol 2012, 41:1337-1346 
18. Yadav H, Quijano C, Kamaraju AK, Gavrilova O, Malek R, Chen W, Zerfas P, Zhigang D, Wright EC, Stuelten C, Sun P, Lonning S, Skarulis M, Sumner AE, Finkel T, Rane SG: Protection from obesity and diabetes by blockade of TGF- $\beta /$ Smad3 signaling. Cell Metab 2011, 14:67-79

19. Lee JI, Wright JH, Johnson MM, Bauer RL, Sorg K, Yuen S, Hayes BJ, Nguyen L, Riehle KJ, Campbell JS: Role of Smad3 in platelet-derived growth factor-C-induced liver fibrosis. Am J Physiol Cell Physiol 2016, 310:C436-C445

20. Duan WJ, Yu X, Huang XR, Yu JW, Lan HY: Opposing roles for Smad2 and Smad3 in peritoneal fibrosis in vivo and in vitro. Am J Pathol 2014, 184:2275-2284

21. Jinno K, Takahashi T, Tsuchida K, Tanaka E, Moriyama K: Acceleration of palatal wound healing in Smad3-deficient mice. J Dent Res 2009, 88:757-761

22. Wang Y, Moges H, Bharucha Y, Symes A: Smad3 null mice display more rapid wound closure and reduced scar formation after a stab wound to the cerebral cortex. Exp Neurol 2007, 203:168-184

23. Ashcroft GS, Yang X, Glick AB, Weinstein M, Letterio JL, Mizel DE, Anzano M, Greenwell-Wild T, Wahl SM, Deng C, Roberts AB: Mice lacking Smad3 show accelerated wound healing and an impaired local inflammatory response. Nat Cell Biol 1999, 1:260-266

24. Dobaczewski M, Bujak M, Li N, Gonzalez-Quesada C, Mendoza LH, Wang XF, Frangogiannis NG: Smad3 signaling critically regulates fibroblast phenotype and function in healing myocardial infarction. Circ Res 2010, 107:418-428

25. Piek E, Ju WJ, Heyer J, Escalante-Alcalde D, Stewart CL, Weinstein M, Deng C, Kucherlapati R, Bottinger EP, Roberts AB: Functional characterization of transforming growth factor beta signaling in Smad2- and Smad3-deficient fibroblasts. J Biol Chem 2001, 276:19945-19953

26. Sato F, Kawamura H, Wu Y, Sato H, Jin D, Bhawal UK, Kawamoto T, Fujimoto K, Noshiro M, Seino H, Morohashi S, Kato Y, Kijima H: The basic helix-loop-helix transcription factor DEC2 inhibits TGF- $\beta$ induced tumor progression in human pancreatic cancer BxPC-3 cells. Int J Mol Med 2012, 30:495-501

27. Kon N, Hirota T, Kawamoto T, Kato Y, Tsubota T, Fukada Y: Activation of TGF-beta/activin signalling resets the circadian clock through rapid induction of Dec1 transcripts. Nat Cell Biol 2008, 10:1463-1469

28. Yang X, Letterio JJ, Lechleider RJ, Chen L, Hayman R, Gu H, Roberts AB, Deng C: Targeted disruption of SMAD3 results in impaired mucosal immunity and diminished $\mathrm{T}$ cell responsiveness to TGF-beta. EMBO J 1999, 18:1280-1291

29. Kohsaka A, Das P, Hashimoto I, Nakao T, Deguchi Y, Gouraud SS, Waki H, Muragaki Y, Maeda M: The circadian clock maintains cardiac function by regulating mitochondrial metabolism in mice. PLoS One 2014, 9:e112811

30. Sato F, Muragaki Y, Zhang Y: DEC1 negatively regulates AMPK activity via LKB1. Biochem Biophys Res Commun 2015, 467: $711-716$
31. Takahashi JS, Hong HK, Ko CH, McDearmon EL: The genetics of mammalian circadian order and disorder: implications for physiology and disease. Nat Rev Genet 2008, 9:764-775

32. Foster RG, Roenneberg T: Human responses to the geophysical daily, annual and lunar cycles. Curr Biol 2008, 18:R784-R794

33. Baier PC, Brzózka MM, Shahmoradi A, Reinecke L, Kroos C, Wichert SP, Oster H, Wehr MC, Taneja R, Hirrlinger J, Rossner MJ: Mice lacking the circadian modulators SHARP1 and SHARP2 display altered sleep and mixed state endophenotypes of psychiatric disorders. PLoS One 2014, 9:e110310

34. Bae K, Jin X, Maywood ES, Hastings MH, Reppert SM, Weaver DR: Differential functions of mPer1, mPer2, and mPer3 in the SCN circadian clock. Neuron 2001, 30:525-536

35. Massagué J: How cells read TGF-beta signals. Nat Rev Mol Cell Biol 2000, 1:169-178

36. Le NQ, Binh NT, Takarada T, Takarada-Iemata M, Hinoi E, Yoneda Y: Negative correlation between Per1 and Sox6 expression during chondrogenic differentiation in pre-chondrocytic ATDC5 cells. J Pharmacol Sci 2013, 122:318-325

37. Sato F, Wu Y, Bhawal UK, Liu Y, Imaizumi T, Morohashi S, Kato Y, Kijima H: PERIOD1 (PER1) has anti-apoptotic effects, and PER3 has pro-apoptotic effects during cisplatin (CDDP) treatment in human gingival cancer CA9-22 cells. Eur J Cancer 2011, 47:1747-1758

38. Qian Y, Jung YS, Chen X: Differentiated embryo-chondrocyte expressed gene 1 regulates p53-dependent cell survival versus cell death through macrophage inhibitory cytokine-1. Proc Natl Acad Sci U S A 2012, 109:11300-11305

39. Liu Y, Sato F, Kawamoto T, Fujimoto K, Morohashi S, Akasaka H, Kondo J, Wu Y, Noshiro M, Kato Y, Kijima H: Anti-apoptotic effect of the basic helix-loop-helix (bHLH) transcription factor DEC2 in human breast cancer cells. Genes Cells 2010, 15:315-325

40. Iwata T, Kawamoto T, Sasabe E, Miyazaki K, Fujimoto K, Noshiro M, Kurihara H, Kato Y: Effects of overexpression of basic helix-loop-helix transcription factor Dec1 on osteogenic and adipogenic differentiation of mesenchymal stem cells. Eur J Cell Biol 2006, 85:423-431

41. Gery S, Komatsu N, Baldjyan L, Yu A, Koo D, Koeffler HP: The circadian gene Per1 plays an important role in cell growth and DNA damage control in human cancer cells. Mol Cell 2006, 22:375-382

42. Nakashima A, Kawamoto T, Honda KK, Ueshima T, Noshiro M, Iwata T, Fujimoto K, Kubo H, Honma S, Yorioka N, Kohno N, Kato Y: DEC1 modulates the circadian phase of clock gene expression. Mol Cell Biol 2008, 28:4080-4092

43. Li Y, Song X, Ma Y, Liu J, Yang D, Yan B: DNA binding, but not interaction with Bmal1, is responsible for DEC1-mediated transcription regulation of the circadian gene mPer1. Biochem J 2004, 382:895-904

44. Hoyle NP, Seinkmane E, Putker M, Feeney KA, Krogager TP, Chesham JE, Bray LK, Thomas JM, Dunn K, Blaikley J, O’Neill JS: Circadian actin dynamics drive rhythmic fibroblast mobilization during wound healing. Sci Transl Med 2017, 9:eaal2774 